\title{
ERRATUM
}

\section{INECO Frontal Screening (IFS): A brief, sensitive, and specific tool to assess executive functions in dementia - ERRATUM}

TERESA TORRALVA, MARÍA ROCA, EZEQUIEL GLEICHGERRCHT, PABLO LÓPEZ AND FACUNDO MANES

doi:10.1017/S1355617709990415, Published by Cambridge University Press, 28 July 2009.

Material from the Wechsler Memory Scale-Revised was included under the heading 'Digits backward' in the Appendix of the article by Torralva et al. (2009), without attribution to the owner of the material. The Editor wishes to apologise for this oversight and to confirm that the said material is an original work of authorship created by Dr David Wechsler and the copyright is owned by NCS Pearson, Inc.
A Corrected Version of Record has been published in its place.

\section{REFERENCE}

Torralva, T., Roca, M., Gleichgerrcht, E., López, P. \& Manes, F. (2009) INECO frontal screening (IFS): a brief, sensitive, and specific tool to assess executive functions in dementia. Journal of the International Neuropsychological Society, 15, 777-786. doi:10.1017/S1355617709990415. 


\title{
INECO Frontal Screening (IFS): A brief, sensitive, and specific tool to assess executive functions in dementia-CORRECTED VERSION
}

\author{
TERESA TORRALVA,,${ }^{1,2}$ MARÍA ROCA, ${ }^{1,2}$ EZEQUIEL GLEICHGERRCHT, ${ }^{1}$ PABLO LÓPEZ,,${ }^{1,2}$ AND \\ FACUNDO MANES ${ }^{1,2}$ \\ ${ }^{1}$ Institute of Cognitive Neurology (INECO), Buenos Aires, Argentina \\ ${ }^{2}$ Institute of Neurosciences Favaloro University, Buenos Aires, Argentina
}

(Received January 1, 2009; Final Revision June 16, 2009; AcCePted June 16, 2009; Original Version Published OnLine July 28, 2009)

\begin{abstract}
Although several brief sensitive screening tools are available to detect cognitive dysfunction, few have been developed to quickly assess executive functioning (EF) per se. We designed a new brief tool to evaluate EF in neurodegenerative diseases. Patients with an established diagnosis of behavioral variant frontotemporal dementia (bvFTD; $n=22$ ), Alzheimer disease (AD; $n=25)$, and controls $(n=26)$ were assessed with a cognitive screening test, the INECO Frontal Screening (IFS), and EF tests. Clinical Dementia Rating Scale (CDR) scores were obtained for all patients. Internal consistency of the IFS was very good (Cronbach's alpha=.80). IFS total (out of 30 points) was $27.4(S D=1.6)$ for controls, $15.6(S D=4.2)$ for bvFTD, and $20.1(S D=4.7)$ for AD. Using a cutoff of 25 points, sensitivity of the IFS was $96.2 \%$, and specificity $91.5 \%$ in differentiating controls from patients with dementia. The IFS correlated significantly with the CDR and executive tasks. The IFS total discriminated controls from demented patients, and bvFTD from AD. IFS is a brief, sensitive, and specific tool for the detection of executive dysfunction associated with neurodegenerative diseases. The IFS may be helpful in the differential diagnosis of FTD and AD. (JINS, 2009, 15, 777-786.)
\end{abstract}

Keywords: Neuropsychology, Cognition, Dysexecution, Frontotemporal dementia, Alzheimer disease, Differential diagnosis

\section{INTRODUCTION}

Executive functioning (EF) refers to the end result of a coordinated sequence of cognitive processes aimed at achieving a particular goal in a flexible manner. The prefrontal cortex is essential for these processes (Funahashi, 2001; Fuster, 1997; Stuss \& Benson, 1986), and several neurological (Cummings, 1993; Graham, Bak, \& Hodges, 2003; Williams-Gray, Foltynie, \& Brayne, 2007; Zakzanis, Leach, \& Freedman, 1998) and psychiatric disorders (Reichenberg et al., 2008; Roth \& Saykin, 2004) are characterized by deficits in EF. However, it is now well established that different pathologies typically classified within the same umbrella, such as various types of dementia, are not equally impaired in this domain. For example, it is known that patients who develop the behavioral variant of frontotemporal dementia (bvFTD) have early and prominent impairments in executive functions due to early changes in frontal lobe structure (for review, see Hodges \& Miller, 2001a,

Correspondence and reprint requests to: Teresa Torralva, Instituto de Neurología Cognitiva (INECO), Castex 3293 (1425), Buenos Aires, Argentina. E-mail: ttorralva@ neurologiacognitiva.org 2001b). On the contrary, patients with Alzheimer's disease (AD), can present very mild executive dysfunction but are not characterized, at least during the early stages, by prominent executive problems. Rather, they tend to present deficits in episodic memory due to early changes in medial temporal lobe structures (for review, see Nestor, Scheltens, \& Hodges, 2004). While an accurate evaluation of executive functions is critical for accurate differential diagnosis of a range of conditions, the detection of a dysexecutive syndrome typically requires an extensive neuropsychological battery. A brief screening tool which is easy to administer, yet shows high sensitivity, specificity, and predictive value would be of great importance to clinicians. Several cognitive screening tools have desirable diagnostic and statistical properties (Cullen, O'Neill, Evans, Coen, \& Lawlor, 2007), but few have been designed to specifically assess executive functioning. As evidence of the intrinsic difficulties that arise with the development of such tools, various screening batteries that have attempted to measure executive dysfunction, fail to exhibit reasonable psychometric properties. For instance, Rothlind and Brandt (1993) proposed a brief screening test for detecting 
frontal and subcortical dysfunction, however, patients with AD showed worse performance on this test than patients with frontal dysfunction showing low specificity of the tool. Royall, Mahurin, and Gray (1992) developed an executive functioning interview reflecting a similar problem, being sensitive also to nonexecutive dysfunction. Ettlin and Kischka developed the Frontal Lobe Score, but administration of this tool requires at least $40 \mathrm{~min}$ (Ettlin \& Kischka, 1999).

The Frontal Assessment Battery (FAB; Dubois, Slachevsky, Litvan, \& Pillon, 2000) is an executive screening test that, over the last years, has become widely used in neurological research (Guedj et al., 2008; Lima, Meireles, Fonseca, Castro, \& Garrett, 2008; Oguro et al., 2006; Santangelo et al., 2009; Yoshida et al., 2009). It consists of six subtests assessing conceptualization, cognitive flexibility, motor programming, sensitivity to interference, motor inhibitory control, and prehension behavior. The authors of the FAB have proposed this test for the evaluation of different kinds of frontal dysfunction, and also to distinguish between neurological pathologies such bvFTD and AD (Slachevsky et al., 2004). They report correlations with executive measures such as the Wisconsin Card Sorting Test (WCST) and measures of general cognitive functioning (Mattis Dementia Scale), while highlighting the lack of correlation with the Mini Mental State Examination (MMSE). The original authors conclude that the FAB is an easy-to-administer battery, sensitive to frontal dysfunction. By contrast, other studies have cast doubts on the sensitivity and specificity of the FAB, and in particular, its ability to actually differentiate types of dementia, such as AD and FTD (Castiglioni et al., 2006; Lipton et al., $2005)$ in the early stages. Moreover, although the original study by Dubois et al. (2000) showed no correlation between the FAB and general cognitive measures, subsequent studies failed to replicate these findings suggesting that performance on the FAB does not reflect frontal function exclusively (Castiglioni et al., 2006; Lipton et al., 2005).

Given the aforementioned difficulties and based on our previous research studies on executive tests (Clark, Manes, Antoun, Sahakian, \& Robbins, 2003; Clark \& Manes, 2004; Manes et al., 2002; Torralva et al., 2007), we designed a tool aimed at detecting executive dysfunction: the INECO Frontal Screening (IFS). This screening test was designed to provide health professionals with a sensitive and specific executive screening test to determine frontal dysfunction in patients with dementia. We incorporated some of the FAB subtests which showed the highest sensitivity in our everyday clinical experience: motor programming, conflicting instructions, and inhibitory control. For various clinical and practical reasons, we refrained from including the following subtests of the FAB: verbal fluency, because it is usually administered in general cognitive screening batteries; similarities, as we included a more complex subtest of conceptualization; and prehension behavior, which would be better regarded as a neurological sign of disinhibition ("grasping sign") than as an index of neuropsychological impairment and is only affected in patients with extremely severe frontal dysfunction (Iavarone et al., 2004). Of the FAB subtests included in the IFS, motor programming has been shown to have the highest sensitivity in a study by Lipton et al. (2005) while conflicting instructions and inhibitory control are, in our experience, two subtests that usually pose difficulties to our frontal patients. To design a more sensitive and specific tool, we also included new subtests that have been shown to be sensitive to executive dysfunction: numerical working memory (backward digit span), verbal working memory (months backward), spatial working memory (modified Corsi tapping test), conceptualization (proverbs), and verbal inhibitory control (modified Hayling test; Burgess \& Shallice, 1997a).

The executive domain encompasses several different functions and the IFS was designed to incorporate a few measures that could tap, in a brief way, as many of these functions as possible. Considering this, the inclusion of the subtests of the IFS was based on the model presented in Table 1 . The design of the IFS was primarily conceived as representing three groups of tasks, as follows: (a) response inhibition and set shifting - evaluates the ability to shift from one cognitive set to another and to inhibit inappropriate response in a verbal and motor way; (b) capacity of abstraction - obtained from proverb interpretation, with concrete interpretation being typical of frontal lobe damage patients; (c) working memory - referring to a brain system that provides temporary storage and manipulation of the information necessary for other complex cognitive tasks, one of the most well known models, proposed by Baddeley and Hitch (1974), has a three component structure including a Central Executive, which is involved in the control and regulation of the Working Memory System, and two "slave systems", one responsible for holding verbal information for short periods (phonological loop) and the other for holding information in visual and spatial form (visuo-spatial sketchpad).

Overall, the goal of this study was to evaluate a new, easy-to-administer, brief (approximately $10 \mathrm{~min}$ ), sensitive,

Table 1. Subtests grouped into the different executive functions tapped by the IFS

$\left.\begin{array}{ll}\hline \hline \text { Executive function } & \multicolumn{1}{c}{\text { IFS subtest }} \\ \hline \begin{array}{c}\text { Response inhibition } \\ \text { and set shifting }\end{array} & \begin{array}{l}\text { Motor programming } \\ \text { Conflicting instructions } \\ \text { Go-No go } \\ \text { Verbal inhibitory control (Modified } \\ \text { Hayling test) } \\ \text { Proverb interpretation } \\ \text { Abstraction }\end{array} \\ \text { Working Memory } & \begin{array}{l}\text { Berbal Working Memory* } \\ \text { Spatial Working Memory** }\end{array}\end{array}\right\} \begin{aligned} & \text { Central } \\ & \text { Executive }\end{aligned}$

Note. IFS = INECO Frontal Screening

*Predominantly verbal (Phonological Loop).

***Predominantly visual (visuo-spatial sketchpad) 
and specific tool for the assessment of executive functioning. We also investigated whether the IFS could discriminate executive functioning between bvFTD and AD. We hypothesized that a screening tool specifically designed to measure executive functioning may differentiate the executive deficits characteristic of frontal damage (bvFTD) from the subtle executive deficits of the early $\mathrm{AD}$, a condition which mainly involves medial temporal lobe structures at early stages. Specifically, we propose that, because of the early and prominent executive impairment of bvFTD, these patients would perform worse than the AD group on said screening tool.

\section{METHODS}

\section{Participants}

A total of 73 participants were included in this study, 26 of which were healthy controls, and 47 of which were diagnosed with dementia. Within the dementia group, 22 patients presented with the bvFTD and 25 with a diagnosis of probable AD. Healthy controls were examined with a comprehensive neuropsychological and neuropsychiatry evaluation, and had no history of either neurological or psychiatric disorder. All patients with AD diagnosis fulfilled NINCDS-ADRDA criteria (Varma et al., 1999), while all patients in the bvFTD group fulfilled Lund and Manchester criteria (Neary et al., 1998). All patients underwent a standard examination battery including neurological, neuropsychiatric, and neuropsychological examinations and a MRI-SPECT. bvFTD patients showed frontal atrophy on MRI, and frontal hypoperfusion on SPECT, when available. Patients with a Clinical Dementia Rating (CDR) Scale of two points or higher were excluded from this study (Hughes, Berg, Danzinger, Coben, \& Martin, 1982). However, to avoid circularity, specialists determining diagnoses of patients included in the analysis were blind to their performance on the tool introduced in this study, the INECO Frontal Screening (IFS).

\section{Procedure}

The study was initially approved by the ethics committee at the Institute of Cognitive Neurology (INECO) following international regulations established for human research subjects. All participants were evaluated with an extensive neuropsychological battery. Data for this study were obtained from the following tests: the IFS, the Addenbrooke's Cognitive Examination (ACE; Mathuranath, Nestor, Berrios, Rakowicz, \& Hodges, 2000) which also incorporates the MMSE (Folstein, Folstein, \& McHugh, 1975), and classical executive measures, including the verbal phonological fluency (Lezak, 1995), Trail Making Test - Part B (Partington \& Leiter, 1949), and the WCST (Nelson, 1976). IFS content, instructions, and scoring for each of the subtests are included as an appendix to this article. Total IFS score is calculated as the sum of each of the eight subtest scores. Overall average administration time is approximately $10 \mathrm{~min}$.

\section{IFS Subtests}

1. Motor Programming (3 points) (Dubois et al., 2000; Luria, 1966). This subtest asks the patient to perform the Luria series, "fist, edge, palm" by initially copying the administrator, and by subsequently doing the series on his or her own then by repeating the series six times alone. Depending on the extent of frontal lesion or degeneration, some patients may not be able to complete the series in the correct order on their own, and others may not even be capable of copying it. If subjects achieved six consecutive series by themselves, the score was 3 , if they achieved at least three consecutive series on their own, the score was 2 ; if they failed at achieving at least three consecutive series alone, but achieved three when copying the examiner, the score was 1 ; otherwise the score was 0 .

2. Conflicting Instructions (3 points) (Dubois et al., 2000). Interference (Dubois et al., 2000). Subjects were asked to hit the table once when the administrator hit it twice, or to hit the table twice when the administrator hit it only once. To ensure the subject had clearly understood the task, a practice trial was performed in which the administrator first hit the table once, three times in succession, and then twice, three more times. After the practice trial, the examiner completed the following series: 1-1-2-1-2-2-2-1-1-2. If subjects made no errors, the score was 3 ; if they made one or two errors, the score was 2; for more than two errors, the score was 1 , unless the subject copied the examiner at least four consecutive times, in which case the score was 0 . Patients with frontal lesions tend to imitate the examiner's movements, ignoring the verbal instruction.

3. Go-No Go (3 points) (Dubois et al., 2000). This task was administered immediately after test 2 . Subjects were told that now, when the test administrator hit the table once, they should hit it once as well, but when the examiner hit twice, they should do nothing. To ensure the subject had clearly understood the task, a practice trial was performed in which the administrator hit the table once, three times in succession, and then twice, three more times. After the practice trial the examiner completed the following series: 1-1-2-1-2-2-2-1-1-2. If subjects made no errors, the score was 3 ; for one or two errors the score was 2; for more than two errors the score was 1 , unless the subject copied the examiner at least four consecutive times, in which case the score was 0 .

4. Backward Digit Span (6 points) (Hodges, 1994). For this task, subjects were asked to repeat a progressively lengthening string of digits in the reverse order. Two trials were 
given at each successive list length, beginning at two and continuing to a maximum of seven. If subjects passed either trial at a given list length, then the next length was administered. The score was the number of lengths at which the subject passed either trial, maximum 6 .

5. Verbal Working Memory (2 points) (Hodges, 1994). The patient was asked to list the months of the year backward, starting with December. If subjects made no errors, the score was 2; for one error, the score was 1; otherwise the score was 0 . This task evaluates the same function as the previous subtest but with a slightly different load because the series is highly overlearned for most individuals.

6. Spatial Working Memory (4 points) (Wechsler, 1987). In this task, the examiner presented the subject with four cubes and pointed at them in a given sequence. The subject was asked to repeat the sequence in reverse order. There were four trials, with sequences of two, three, four, and five cubes respectively. Score was number of correctly completed sequences.

7. Abstraction Capacity (Proverb interpretation) (3 points) (Hodges, 1994). Patients with frontal lesions show difficulties in abstract reasoning tasks. Reasoning is most frequently clinically assessed in one of two ways, namely, with either similarities or proverb interpretation tasks. The latter was chosen for this screening test, because patients with frontal lesions usually have difficulties in stepping away from the concrete facts to find their abstract meaning. In this task, three proverbs were read to the subjects and they were asked to explain their meaning. For each proverb a score of 1 was given when the subject gave an adequate explanation, and a score of 0.5 for a correct example. Otherwise the score was 0 . The three proverbs were chosen specifically for this demographic population based on their high frequency in oral speech.

8. Verbal Inhibitory Control (6 points) (Burgess \& Shallice, 1997b). This task, inspired by the Hayling test, measures a subject's capacity to inhibit an expected response. Materials were six sentences, each missing the last word and constructed to strongly constrain what it should be. In the first part (three sentences), subjects were read each sentence and asked to complete it correctly, as quickly as possible. In the second part (remaining three sentences), subjects were asked for a completion that was syntactically correct but unrelated to the sentence in meaning. Only the second part was scored. For each sentence, a score of 2 was given for a word unrelated to the sentence, a score of 1 for a word semantically related to the expected completion, and a score of 0 for the expected word itself. Example: "An eye for an eye, a tooth for a ...(table)..." By presenting an identical structure during both phases, this subtest is potentially capable of efficiently evaluating two executive function components (initiation and inhibition) in relation to a unique symbolic verbal form (Abusamra, Miranda, \& Ferreres, 2007).

\section{Statistical Analysis}

Internal consistency was determined with Cronbach's alpha coefficient. To analyze concurrent validity with other tasks shown to be sensitive to damage to the prefrontal cortex, the IFS total score was correlated with the Clinical Dementia Rating (CDR) Scale; the ACE total score; the MMSE; the number of items produced on the phonological fluency task, the number of categories abstracted, and perseverative errors on the WCST; and latency to complete Part B of the Trail Making Test (TMT-B).

The ability of the IFS to discriminate healthy controls from patients diagnosed with either form of dementia included in our study (AD or bvFTD) was determined using a receiver operating characteristic (ROC) curve analysis. Demographic and clinical information, as well as neuropsychological test performance were compared between the groups using oneway analyses of variance with Bonferroni post hoc analyses when appropriate. When data were not normally distributed, Mann-Whitney $U$ tests were used to compare two groups at a time. When analyzing categorical variables (e.g., gender), the Freeman-Halton extension of the Fisher exact probability test for $2 \times 3$ contingency tables was used. Inter-rater reliability was determined using Cohen's kappa coefficient by two independent raters (T.T. \& M.R.). All statistical analyses were performed using the SPSS 15.0 software package.

\section{RESULTS}

\section{Clinical and Cognitive Profile}

Demographic profile and total scores on tests of general cognitive status are summarized in Table 2 . A significant difference was found for age $\left(F_{2,72}=10.4 ; p<.001\right)$, with $\mathrm{AD}$ patients differing from both controls $(p<.001)$ and bvFTD patients $(p<.01)$. Nonetheless, neither years of formal education $\left(F_{2,72}=2.63 ; p=.082\right)$ nor gender $\left(\chi^{2}=0.25\right.$; $p=.88)$ differed significantly between the groups. As expected, significant differences were found for the CDR $\left(\mathrm{F}_{2,72}=91.7 ; p<.001\right)$, with controls scoring significantly lower than the dementia groups (both, $p<.001$ ), but no differences between $\mathrm{AD}$ and FTD $(p=.91)$. The MMSE $\left(\mathrm{F}_{2,72}=60.5 ; p<.001\right)$ and the ACE $\left(\mathrm{F}_{2,72}=48.3 ; p<.001\right)$ differed across the groups (all comparisons, $p<.01$ ).

Table 2. Demographic and general cognitive status information

\begin{tabular}{lccc}
\hline \hline & bvFTD $(n=22)$ & $\mathrm{AD}(n=25)$ & Control $(n=26)$ \\
\hline Age & $70.5(6.1)$ & $77.6(5.2)$ & $69.2(8.9)$ \\
Years of education & $16.3(3.1)$ & $14.5(3.6)$ & $14.5(2.2)$ \\
Gender (M : F) & $9: 13$ & $12: 13$ & $12: 14$ \\
MMSE & $27.6(2.1)$ & $24.4(2.6)$ & $29.6(0.4)$ \\
ACE & $80.4(11.5)$ & $68.6(9.9)$ & $95.5(3.1)$ \\
CDR & $0.85(0.46)$ & $0.93(0.17)$ & 0 \\
\hline \hline
\end{tabular}

Note. Values are expressed as Mean $(S D)$. bvFTD=behavioral variant frontotemporal dementia; $\mathrm{AD}=$ Alzheimer's disease; $\mathrm{MMSE}=$ Mini Mental State Examination; $\mathrm{ACE}=$ Addenbrooke's Cognitive Examination; $\mathrm{CDR}=\mathrm{Clinical}$ Dementia Rating Scale. 


\section{Psychometric Properties}

Internal consistency of the IFS was very good (Cronbach's alpha $=0.80$ ), and most subtests correlated significantly between themselves (Table 3 ). Inter-rater reliability was very good (Cohen's kappa $=0.87$ ). IFS total score was 27.4 $(S D=1.6)$ for controls, $15.6(S D=4.2)$ for bvFTD, and 20.1 $(S D=4.7)$ for AD. A significant difference was found between the groups on the IFS total score $\left(F_{2,72}=63.7 ; p<\right.$ .001 ), as controls performed significantly better than both bvFTD $(p<.001)$ and $\operatorname{AD}(p<.001)$ patients. Moreover, both dementia groups differed significantly between themselves on the IFS total score $(p<.001)$, as shown by Figure 1 .

The IFS total score also correlated (Figure 2) with classical executive tests: the number of items produced on the phonological fluency task $(r=0.67, p<.001)$, the total number of categories abstracted $(r=0.77 ; p<.001)$ and perseverative errors $(r=-0.77 ; p<.001)$ on the WCST, and time to complete the TMT-B $(r=-0.75 ; p<.001)$. Interestingly, when correlations were calculated exclusively within the bvFTD group, no significance was found between the IFS total score and the MMSE $(r=.17 ; p=.45)$, ACE $(r=.09 ; p=.67)$, or the CDR $(r=-.16$; $p=.50$ ), as shown by the black-filled dots on Figure 2 correlation graphs. However, when correlations were calculated exclusively within the $\mathrm{AD}$ group, significant correlations were found between the IFS total score and the MMSE $(r=.57 ; p<.01)$, $\operatorname{ACE}(r=.55 ; p<.01)$. A similar profile was observed within the control group (MMSE: $r=.40 ; p=.04$; ACE: $r=.55 ; p<.01$ ).

To demonstrate the specificity of IFS to executive functioning, further correlations were conducted within the bvFTD group between IFS total score and the subscores of the ACE domains. While a significant correlation was found with the attention domain $(r=.59 ; p<.05)$ as expected, no significant correlations were found with orientation $(r=.04 ; p=.87)$,

Table 3. Inter-subscale correlation matrix

\begin{tabular}{lllllrrrrr}
\hline \hline (A) & & MP & CI & MIC & BDS & VWM & SWM & AC & VIC \\
\hline MP & $r$ & $* *$ & -.02 & .29 & .29 & .20 & .22 & .45 & .42 \\
& $p$ & & .89 & .01 & .01 & .09 & .06 & .01 & $<.01$ \\
CI & $r$ & & $* *$ & .17 & .17 & .01 & .09 & .13 & .10 \\
& $p$ & & & .15 & .16 & .99 & .45 & .27 & .41 \\
MIC & $r$ & & & $* *$ & .47 & .49 & .41 & .55 & .50 \\
& $p$ & & & $<.01$ & $<.01$ & $<.01$ & $<.01$ & $<.01$ \\
BDS & $r$ & & & & $* *$ & .39 & .43 & .59 & .51 \\
& $p$ & & & & $<.01$ & $<.01$ & $<.01$ & $<.01$ \\
VWM & $r$ & & & & $* *$ & .24 & .44 & .34 \\
& $p$ & & & & & .04 & $<.01$ & $<.01$ \\
SWM & $r$ & & & & & $* *$ & .43 & .38 \\
& $p$ & & & & & & $<.01$ & $<.01$ \\
AC & $r$ & & & & & & $* *$ & .60 \\
& $p$ & & & & & & & $<.01$ \\
VIC & $r$ & & & & & & & \\
& $p$ & & & & & & & & \\
\hline
\end{tabular}

Note. $\mathrm{MP}=$ Motor Programming; $\mathrm{CI}=$ Conflicting Instructions; $\mathrm{MIC}=\mathrm{Mo}-$ tor Inhibitory Control; BDS = Backwards Digit Span; VWM = Visual Working Memory; SWM = Spatial Working Memory; $\mathrm{AC}=$ Abstraction Capacity; VIC = Verbal Inhibitory Control.

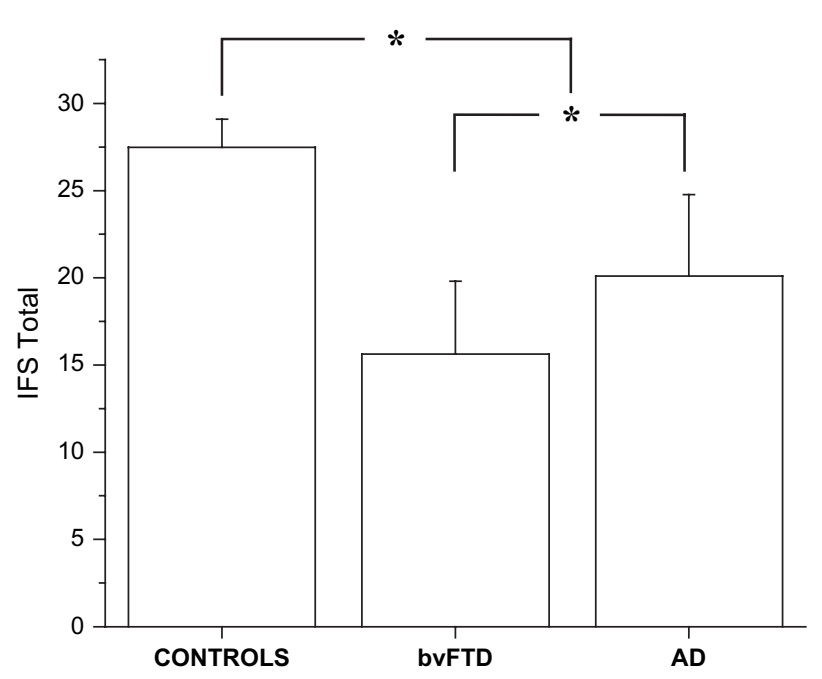

Fig. 1. Mean (SD) scores for Controls, Alzheimer's disease (AD), and behavioral variant frontotemporal dementia (bvFTD) groups on the INECO Frontal Screening (IFS).

memory $(r=.05 ; p=.84)$, fluency $(r=.40 ; p=.09)$, language $(r=.18 ; p=.43)$, or visuo-construction $(r=.38 ; p=.09)$.

A detailed comparison of $\mathrm{AD}$ versus bvFTD performance on each of the eight IFS subtests (Figure 3) revealed that the groups differed significantly on the motor inhibitory control, Go-No Go task $(U=182.0 ; p=.038)$, the verbal working memory task $(U=174.5 ; p=.014)$, the abstraction capacity task $(U=113.5 ; p<.001)$, and the verbal inhibitory control Hayling test $(U=144.0 ; p<.001)$.

A ROC curve analysis on the IFS total score between healthy controls and patients (AD and bvFTD) generated a cutoff score of 25 points with sensitivity of $96.2 \%$ and specificity of $91.5 \%$ (Figure 4). Area under the ROC curve was .98 (CI: .95-1.04; $p<.001$ ). Furthermore, when patient groups were separated based on their form of dementia, a ROC curve analysis between both groups generated a cutoff score of 19 points, with a sensitivity of $72.0 \%$ and a specificity of $81.3 \%$, with a smaller, yet significant $(p<.01)$ area under the curve of .776 (CI: .62-.90). With an IFS cutoff score of $26,100 \%$ of the bvFTD patients were detected as bearing the executive deficits expected for frontotemporal dementia versus $12 \%$ of controls. In contrast, the 88-point cutoff score set by the ACE detected $63.4 \%$ of the bvFTD patients and the 23 cutoff score set by the MMSE detected only $4.6 \%$ of the bvFTD patients. To further analyze the superior sensitivity of the IFS in differentiating bvFTD patients from $\mathrm{AD}$, the area under the curve $(\mathrm{AuC})$ of the classical executive tasks was compared with the $\mathrm{AuC}$ of the IFS. While, as previously stated, the latter was significant $(p<.001)$, the executive tasks had poor discrimination accuracy (Phonological fluency: $\mathrm{AuC}=.487, p=.89$; WCST: $\mathrm{AuC}=.618, p=.36$; TMT-B: $\mathrm{AuC}=.464, p=.71$ ).

\section{DISCUSSION}

In our study, the IFS has demonstrated good psychometric properties: very good internal consistency; excellent 

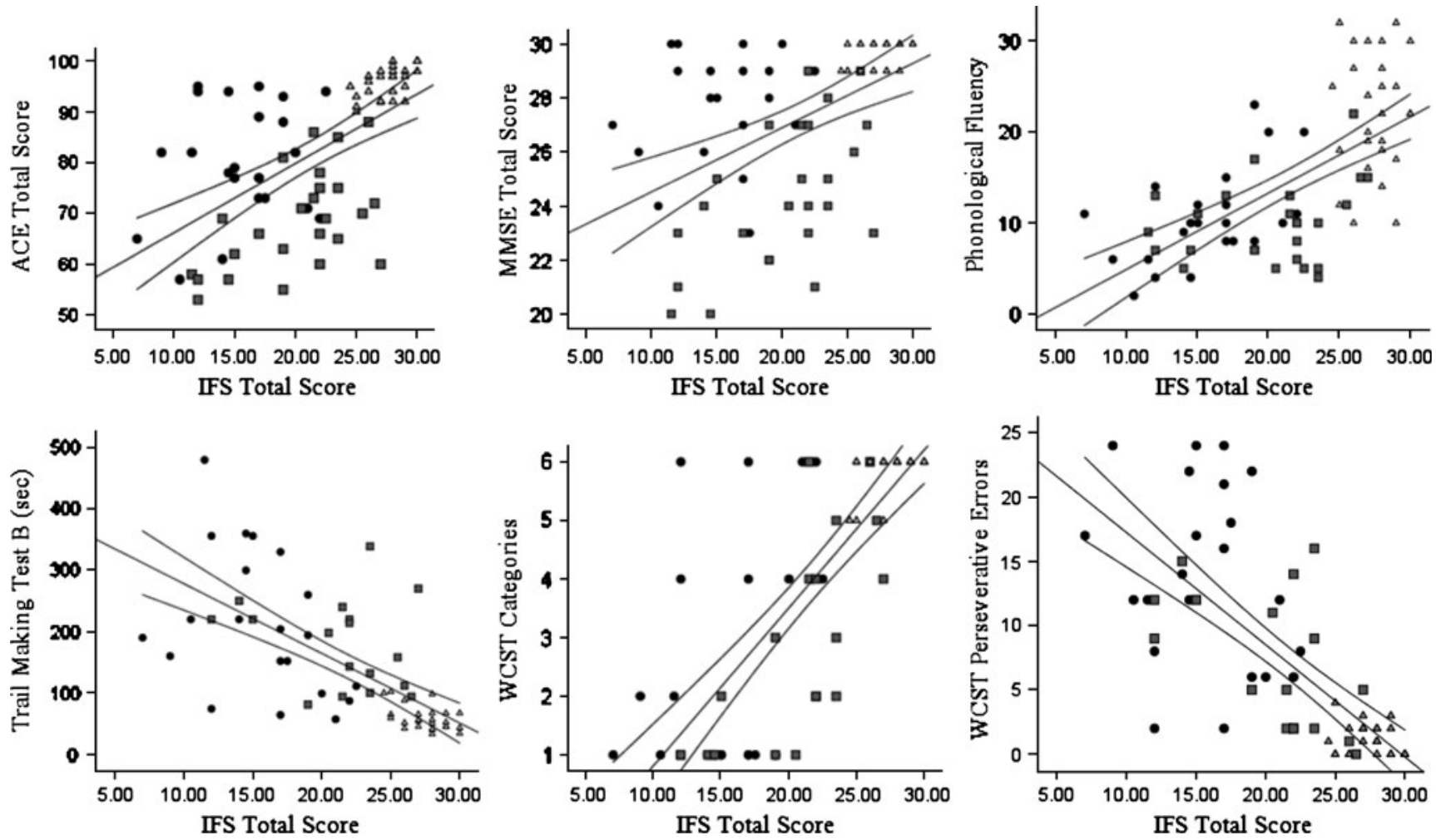

Fig. 2. Correlations between the INECO Frontal Screening (IFS) total score and the Mini Mental State Examination (MMSE), the Addenbrooke's Cognitive Examination (ACE) total score, phonological fluency task, latency to complete Trail Making Test-Part B (TMT-B), and Wisconsin Card Sorting Test (WCST, categories and perseverations). Data points represent behavioral variant frontotemporal dementia (bvFTD, •), Alzheimer's disease (AD, $\boldsymbol{\square})$, and controls $(\boldsymbol{\Delta})$.

Linear fit lines are presented with mean confidence intervals.

concurrent validity, as shown by its significant correlation with classical measures of frontal functioning (phonological verbal fluency, WCST categories and perseverative errors, and TMT-B); and good discriminant validity, as revealed by the capability of the IFS to significantly differentiate healthy controls from demented patients, and, furthermore, patients with AD from patients with bvFTD.

One of the most reliable findings of the utility of the IFS is the concurrent validity demonstrated between this test and some of the most classical executive tests available. Several studies have shown the close association between neuropsychological tasks such as the WCST, verbal fluency tasks, and Trail Making Test B, and functioning of the prefrontal cortex. Stuss and Levine (2002) reported a series of studies where the inclusion of the WCST as a "frontal measure" in neuropsychological batteries was strongly justified, describing frontal activation during the execution of this task. In this same way, the phonological verbal fluency task is traditionally considered to be capable of reflecting left frontal functioning in particular (Milner, 1971), and the time performance of the Trail Making Test-Part B is sensitive to frontal pathology (Stuss et al., 2001). The excellent correlations found between these well-established frontal tests and the IFS demonstrate a close association between the total IFS score and executive dysfunction in our groups of patients. Moreover, the weak correlations found between the IFS total score and all subdomains of the ACE (except attention), shows that the concurrent validity of the IFS is highly specific for

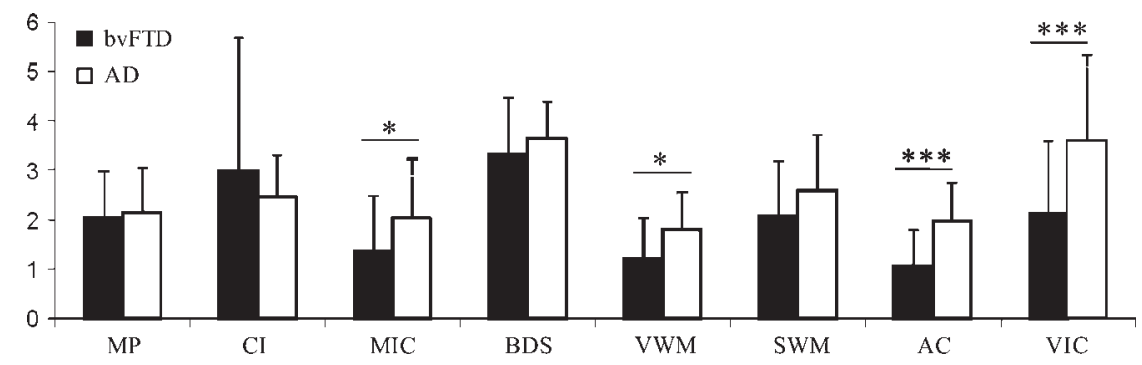

Fig. 3. Mean (SD) scores for Alzheimer's disease (AD) and behavioral variant frontotemporal dementia (bvFTD) groups on each subtests of the INECO Frontal Screening (IFS). ${ }^{*} p<.05, * * * p<.001$. 


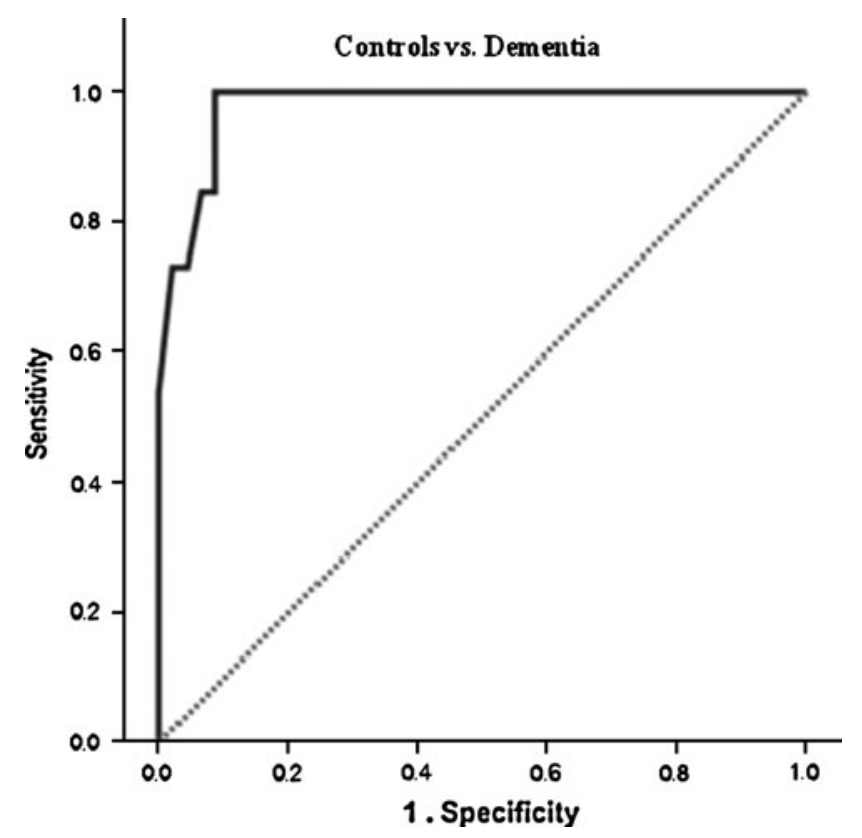

Fig. 4. Receiver operating characteristic (ROC) curve analyses for dementia versus controls (left) and between dementia groups (right).

executive functions. The correlations were, as expected, moderate for the fluency and visuo-spatial domains, because of the relatively strong executive component inherent to these tasks of the ACE (phonological fluency, cube copying, and clock drawing). The high specificity for executive functions demonstrated by the IFS is further supported by the larger accuracy in differentiating AD from bvFTD, as revealed by the analysis of the areas under the curve. In fact, the global IFS score was able to differentiate between bvFTD and AD patients, as well as between both pathological groups and healthy controls. More specifically, bvFTD patients exhibited a more severe executive dysfunction, represented by their lower overall IFS scores compared with patients with AD. This difference becomes especially important when considering that the FAB's initially reported discriminant ability failed to be replicated by other research groups (Castiglioni et al., 2006; Lipton et al., 2005). The more severe executive dysfunction observed in patients with the bvFTD is consistent with the etiological properties of this condition: predominant frontal atrophy and executive disorders since the early stages (Hodges, 2001).

In analyzing each IFS subtest independently, significant differences were found between $\mathrm{AD}$ and bvFTD patients on four particular tasks: Go-No Go, Verbal Working Memory, Proverbs, and the Hayling test. In addition, all subtests (except the conflicting instructions task) showed a clear trend toward a similar profile. The different profile observed for the conflicting instruction task, for which $\mathrm{AD}$ patients showed a worse performance than the bvFTD group requires further exploration, as it may be resulting from high variability within the group, differences in the clinical profiles, or differential performance on other cognitive domains, such as attention.
There are some limitations to this study. First, a significant difference was found between AD and both bvFTD and controls for their mean age. While this is important because of the potential effect of ageing on executive performance, two findings present evidence against this. On one hand, there were no significant differences on the levels of education between the groups, a variable that could have even a stronger effect than age on executive dysfunction. On the other hand, AD patients outperformed bvFTD on all except one subtest of the IFS, showing that, even though the former group was older, IFS still captured the dysexecution of a younger yet selectively impaired bvFTD group. In fact, the differences on age between the groups results from earlier onset of bvFTD in comparison to AD, and highlights the inclusion of early-stage patients in the present study. Another limitation has to do with the relatively small sample sizes used in this study. Naturally, future studies should replicate the present work to strengthen the generalizability of the results.

The ACE is a screening tool that has demonstrated to have excellent specificity and sensitivity for patients with dementia, especially for Alzheimer disease (Mathuranath et al., 2000). One of the most remarkable limitations of the ACE, acknowledged even by its original authors, is its poor capability for the detection of executive dysfunction. We suggest that the administration of both IFS and ACE in the detection of dementia will help overcome this limitation. In this respect, while significant correlations were found on the whole sample (patients and controls) between the IFS total score and general cognitive measures such as the MMSE and the ACE, those correlations were not observed when we split the sample based on their type of dementia. As it can be observed in Figure 2, within the bvFTD group, no significant correlations were found between the IFS total score and global cognitive measures. It is unlikely that this lack of correlation stems from ceiling effects. Unlike controls, most bvFTD patients exhibit high performance on the ACE but poor performance on the IFS. This is especially important because it highlights the fact that the IFS may be specifically capturing executive domains, otherwise undetected by general cognitive tests. In contrast, when correlations were calculated exclusively within the AD group, significant correlations were indeed found between the IFS total score and the MMSE and the ACE. This could be explained by the fact that, as one could expect, the general cognitive status has a direct impact on IFS performance. In fact, to perform within normal ranges on the IFS, preserved cognitive functioning is needed. It is likely that some patients fail to exhibit high performance on a domain-specific task (IFS) if cognitive areas such as comprehension, language, visuo-spatial abilities, and attention, all of which are captured by ACE, are not minimally spared. Moreover, when looking at the individual performances on ACE and IFS within the bvFTD group, patients with low general cognitive functioning (lower scores on ACE) also perform more poorly on the IFS, supporting the idea that minimal general functioning is needed for proper performance on this battery. The lack of correlations found 
between the IFS and both the MMSE and ACE within the control group may be due to ceiling effects, as revealed by Figure 2. Further studies are needed to explore the different profiles within various types of dementia and control populations.

As previously described, we believe that a combination of the ACE and the IFS will maximize the power of early detection of pathologies involving frontal circuitry in highly demanding clinical settings, where a complete neuropsychological evaluation may not be possible, or when assessment time is limited. Further research is needed to determine the assumption that a combination of the ACE and the IFS will increase the efficiency in differentiating types of dementia, and to determine the utility of the IFS in other neurological and psychiatric diseases. Moreover, future research should also explore behavioral observations during assessment of the IFS as alternative and complimentary tools to differentiate $\mathrm{AD}$ from bvFTD patients (e.g., time to complete tasks, latency to respond, etc.)

In summary, although the complexity of executive functions makes it impossible to think of a single test capable of evaluating this cognitive process in its entirety, the present study indicates that the IFS is a solid, brief, and easy-toadminister diagnostic tool for the assessment of executive functions in bvFTD and AD.

\section{ACKNOWLEDGMENTS}

We would like to thank Lucía Crivelli for her valuable contributions to the discussion of the test design. We would also like to thank Catalina Raimondi and Ana Bonifacio for their assistance in this study, as well as Sandra Weintraub for her helpful comments. This study was funded by a FINECO grant and a Fundación LyD grant.

\section{REFERENCES}

Abusamra, V., Miranda, M.A., \& Ferreres, A. [Evaluación de la iniciación e inhibición verbal en español. Adaptación y normas del test de Hayling]. Revista Argentina de Neuropsicología, 9, 19-32.

Baddeley, A.D., \& Hitch, G. (1974). Working memory. In G.H. Bower (Ed.), The psychology of learning and motivation: Advances in research and theory (Vol. 8, pp. 47-89). New York: Academic Press.

Burgess, P.W., \& Shallice, T. (1997b). The Hayling and Brixton Tests. Thurston, Suffolk: Thames Valley Test Company.

Burgess, P., \& Shallice, T. (1997a). The Hayling Test. Northern Speech Services \& National Rehabilitation Services Inc.

Castiglioni, S., Pelati, O., Zuffi, M., Somalvico, F., Marino, L., Tentorio, T., et al. (2006). The Frontal Assessment Battery does not differentiate frontotemporal dementia from Alzheimer disease. Dementia and Geriatric Cognitive Disorders, 22, 125-131.

Clark, L., \& Manes, F. (2004). Social and emotional decisionmaking following frontal lobe injury. Neurocase, 10, 398-403.

Clark, L., Manes, F., Antoun, N., Sahakian, B.J., \& Robbins, T.W. (2003). The contributions of lesion laterality and lesion volume to decision-making impairment following frontal lobe damage. Neuropsychologia, 41, 1474-1183.

Cullen, B., O’Neill, B., Evans, J.J., Coen, R.F., \& Lawlor, B.A. (2007). A review of screening tests for cognitive impairment. Journal of Neurology, Neurosurgery, and Psychiatry, 78, 790799.

Cummings, J.L. (1993). Frontal-subcortical circuits and human behavior. Archives of Neurology, 50, 873-880.

Dubois, B., Slachevsky, A., Litvan, I., \& Pillon, B. (2000). The FAB: A frontal assessment battery at bedside. Neurology, 55, 1621-1626.

Ettlin, T., \& Kischka, U. (1999). Bedside frontal lobe testing. The "frontal lobe score". In B.L. Miller \& J.L. Cummings (Eds.), The human frontal lobes. New York, NY: The Guilford Press.

Folstein, M.F., Folstein, S.E., McHugh, P.R. (1975). "Mini-mental state". A practical method for grading the cognitive state of patients for the clinician. Journal of Psychiatric Research, 12, 189-198.

Funahashi, S. (2001) Neuronal mechanism of executive control by the prefrontal cortex. Neuroscience Research, 39, 147-165.

Fuster, J.M. (1997). The prefrontal cortex (3rd ed.) New York: Raven Press.

Graham, N., Bak, T., \& Hodges, J.R. (2003). Corticobasal degeneration as a cognitive disorder. Movement Disorders, 18, 1224 1232.

Guedj, E., Allali, G., Goetz, C., Le Ber, I., Volteau, M., Lacomblez, L., et al. (2008). Frontal Assessment Battery is a marker of dorsolateral and medial frontal functions: A SPECT study in frontotemporal dementia. Journal of the Neurological Sciences, 273, 84-87.

Hodges, J.R. (1994). Cognitive assessment for clinicians. Oxford: Oxford University Press.

Hodges, J.R. (2001). Frontotemporal dementia (Pick's disease): Clinical features and assessment. Neurology, 56, S6-S10.

Hodges, J.R., \& Miller, B. (2001a). The neuropsychology of frontal variant frontotemporal dementia and semantic dementia. Introduction to the special topic papers: Part II. Neurocase, 7, 113121.

Hodges, J.R., \& Miller, B. (2001b). The classification, genetics and neuropathology of frontotemporal dementia. Introduction to the special topic papers: Part I. Neurocase, 7, 31-35.

Hughes, C.P., Berg, L., Danzinger, W.L., Coben, L.A., \& Martin, R.L. (1982). A new clinical scale for the staging of dementia. British Journal of Psychiatry, 140, 566-572.

Iavarone, A., Ronga, B., Pellegrino, L., Loré, E., Vitaliano, S., Galeone, F., et al. (2004). The Frontal Assessment Battery (FAB): Normative data from an Italian sample and performances of patients with Alzheimer's disease and frontotemporal dementia. Functional Neurology, 19, 191-195.

Lezak, M.D. (1995). Neuropsychological assessment. Oxford, UK: Oxford University Press.

Lima, C.F., Meireles, L.P., Fonseca, R., Castro, S.L., \& Garrett, C. (2008). The Frontal Assessment Battery (FAB) in Parkinson's disease and correlations with formal measures of executive functioning. Journal of Neurology, 255, 1756-1761.

Lipton, A.M., Ohman, K.A., Womack, K.B., Hynan, L.S., Ninman, E.T., \& Lacritz, L.H. (2005). Subscores of the FAB differentiate frontotemporal lobar degeneration from AD. Neurology, 65, 726-731.

Luria, A.R. (1966). Higher cortical function in man. London: Tavistock. 
Manes, F., Sahakian, B., Clark, L., Rogers, R., Antoun, N., Aitken, M., et al. (2002). Decision-making processes following damage to the prefrontal cortex. Brain, 125, 624-639.

Mathuranath, P.S., Nestor, P.J., Berrios, G.E., Rakowicz, W., \& Hodges, J.R. (2000). A brief cognitive test battery to differentiate Alzheimer's disease and frontotemporal dementia. Neurology, $55,1613-1620$.

Milner, B. (1971). Interhemispheric differences in the localization of psychological processes in man. British Medical Bulletin, 27, 272-277.

Neary, D., Snowden, J.S., Gustafson, L., Passant, U., Stuss, D., Black, S., et al. (1998). Frontotemporal lobar degeneration: A consensus on clinical diagnostic criteria. Neurology, 51, 1546-1554.

Nelson, H.E. (1976). A modified card sorting test sensitive to frontal lobe deficits. Cortex, 12, 313-324.

Nestor, P.J., Scheltens, P., \& Hodges, J.R. (2006). Advances in the early detection of Alzheimer's disease. Nature Medicine, 10, S34-S41.

Oguro, H., Yamaguchi, S., Abe, S., Ishida, Y., Bokura, H., \& Kobayashi, S. (2006). Differentiating Alzheimer's disease from subcortical vascular dementia with the FAB test. Journal of Neurology, 253, 1490-1494.

Partington, J.E., \& Leiter, R.G. (1949). Partington's Pathway Test. The Psychological Center Bulletin, 1, 9-20.

Reichenberg, A., Harvey, P.D., Bowie, C.R., Mojtabai, R., Rabinowitz, J., Heaton, R.K., et al. (2008). Neuropsychological function and dysfunction in schizophrenia and psychotic affective disorders. Schizophrenia Bulletin, [Epub ahead of print].

Roth, R.M., \& Saykin, A.J. (2004). Executive dysfunction in attention-deficit/hyperactivity disorder: Cognitive and neuroimaging findings. The Psychiatric Clinics of North America, 27, 83-89.

Rothlind, J.C., \& Brandt, J. (1993). A brief assessment of frontal and subcortical functions in dementia. Journal of Neuropsychiatry and Clinical Neurosciences, 5, 73-77.

Royall, D.R., Mahurin, R.K., \& Gray, K.F. (1992). Bedside assessment of executive cognitive impairment: The executive interview. Journal of American Geriatrics Society, 40, 1221-1226.
Santangelo, G., Vitale, C., Trojano, L., Verde, F., Grossi, D., \& Barone, P. (2009) Cognitive dysfunctions and pathological gambling in patients with Parkinson's disease. Movement Disorders, 24, 899-905.

Slachevsky, A., Villalpando, J.M., Sarazin, M., Hahn-Barma, V., Pillon, B., \& Dubois, B. (2004). Frontal Assessment Battery and differential diagnosis of frontotemporal dementia and Alzheimer disease. Archives of Neurology, 61, 1104-1107.

Stuss, D.T., \& Benson, D.F. (1986). The frontal lobes. New York: Raven Press.

Stuss, D.T., Bisschop, S.M., Alexander, M.P., Levine, B., Katz, D., \& Izukawa, D. (2001). The Trail Making Test: A study in focal lesion patients. Psychological Assessment, 13, 230239.

Stuss, D.T., \& Levine, B. (2002). Adult clinical neuropsychology: Lessons from studies of the frontal lobes. Annual Review of Psychology, 53, 401-433.

Torralva, T., Kipps, C.M., Hodges, J.R., Clark, L., Bekinschtein, T., Roca, M., et al. (2007). The relationship between affective decision-making and theory of mind in the frontal variant of frontotemporal dementia. Neuropsychologia, 45, 342-349.

Varma, A.R., Snowden, J.S., Lloyd, J.J., Talbot, P.R., Mann, D.M., \& Neary, D. (1999). Evaluation of the NINCDS-ADRA criteria in the differentiation of Alzheimer disease and frontotemporal dementia. Journal of Neurology, Neurosurgery, and Psychiatry, 66, 184-188.

Wechsler, D. (1987). Wechsler Memory Scale-Revised. New York: Psychological Corporation.

Williams-Gray, C.H., Foltynie, T., \& Brayne, C. (2007). Evolution of cognitive dysfunction in an incident Parkinson's disease cohort. Brain, 130, 1787-1798.

Yoshida, H., Terada, S., Sato, S., Kishimoto, Y., Ata, T., Ohshima, E., et al. (2009). Frontal assessment battery and brain perfusion imaging in early dementia. Dementia and Geriatric Cognitive Disorders, 27, 133-138.

Zakzanis, K., Leach, L., \& Freedman, M. (1998). Structural and functional meta-analytic evidence for fronto-subcortical system deficit in progressive supranuclear palsy. Brain and Cognition, 38, 283-296.

\section{APPENDIX}

The following is a translated version of the original Spanish language screening tool used on the patients included in this study.

\section{Motor Series (Programming)}

"Look carefully at what I'm doing". The examiner repeats Luria's series "fist, side, palm" three times. "Now you make the same with your right hand, first with me, then by yourself." The examiner repeats the series 3 times with the patient and then says "Now, do it all by yourself".

Scoring: 6 consecutives series alone: 3 / At least 3 consecutive series alone: 2 / Patient fails at 1 but achieves 3 consecutive series with examiner: 1 / Patient does not achieve 3 series with examiner: 0

\section{Conflicting instructions (Interference sensitivity)}

"Hit the desk twice when I hit it once". To ensure the patient has clearly understood the task, hit once on the table, repeat three times: 1-1-1. "Hit the desk once when I hit it twice". To ensure the patient has clearly understood the task, hit twice on the table, repeat three times: 2-2-2. The examiner completes this series: 1-1-2-1-2-2-2$1-1-2$.

Scoring: No errors: 3 / One or two errors: 2 / More than two errors: 1 Patient hits like examiner 4 consecutive times: 0

\section{Go-No Go (Inhibitory Control)}

"Hit the desk once when I hit it once". To ensure the patient has clearly understood the task, hit once on the table, repeat three times: 1-1-1. "Do not hit the desk when I hit it twice". To ensure the patient has clearly understood the task, hit twice on the table, repeat three times: 2-2-2. The examiner completes this series: 1-1-21-2-2-2-1-1-2. 
Scoring: No errors: 3 / One or two errors: 2 / More than two errors: $1 /$ Patient hits like examiner 4 consecutive times: 0

\section{$/ 3$}

\section{Digits backward}

Read each series of numbers out load, at a speed of one word/ second. Ask the patient to repeat the series in the reverse order. Move on to the next task when the patient gets both items on the line wrong.

Scoring: Line is considered correct when the patient gets one or both items correct. Score is the last line achieved correctly.

Material from the Wechsler Memory Scale-Revised was included under the heading 'Digits backward' in the Appendix of this paper without attribution to the owner of the material. The Editor wishes to apologise for this oversight and to confirm that the said material is an original work of authorship created by Dr David Wechsler and the copyright is owned by NCS Pearson, Inc.

\section{6}

\section{Verbal working memory}

Months backwards: (errors are considered if: wrong order, omissions, inconclusive task)

Instruction: Say the months of the year backwards, starting with the last month of the year

Dec - Nov - Oct - Sep - Aug - Jul - Jun - May - April March - Feb - Jan.

$(0$ errors $=2,1$ error $=1,>2$ errors $=0)$

\section{Spatial Working Memory}

"I will point at the squares in a given order. I want you to point them in the reverse order"; patient must copy the se- quence in the reverse order. Do it slowly; patient chooses hand of preference.
a. $1-2$
b. $2-4-3$
c. $3-4-2-1$
d. $1-4-2-3-4$
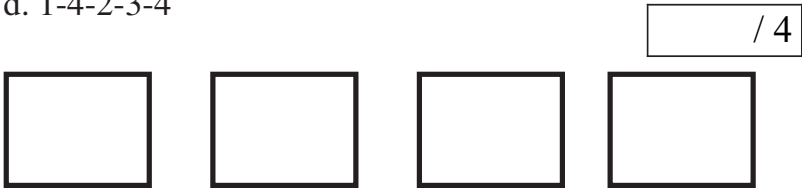

Proverbs: Example: .5 points. Correct explanation: 1 Point

1. "One swallow does not make a summer"

2. "Still waters run deep"

3. "A bird in the hand is worth two in the bush"

\section{Hayling Test (Abbreviated)}

Phase 1: Initiation: "Listen carefully to these sentences and as soon as I am done reading them, you must tell me, as quickly as possible, what word completes the sentence".

I put my shoes on, and I tie my ... (laces)

It was raining cats and ... (dogs)

Phase 2: Inhibition. Different word: 2 / Semantic Relation: 1 / Exact word: 0 point.

"This time, I want you to tell me a word that makes no sense whatsoever in the context of the sentence, and it must not be related to the word that actually completes the sentence."

"For example: Daniel hit the nail with a...rain".

1. John bought candy at the

2. An eye for an eye, a tooth for a

3. I washed my clothes with water and 